\title{
Finite Cell Method for functionally graded materials based on V-models and homogenized microstructures
}

\section{CURRENT STATUS: UNDER REVIEW}

Advanced Modeling and Simulation in Engineering Sciences $\underline{\underline{\underline{Q}} \text { Springer }}$

Benjamin Wassermann

Technische Universitat Munchen

benjamin.wassermann@tum.deCorresponding Author

ORCiD: https://orcid.org/0000-0002-6504-6806

Nina Korshunova

Technische Universitat Munchen

Stefan Kollmannsberger

Technische Universitat Munchen

Ernst Rank

Technische Universitat Munchen

Gershon Elber

Technion Israel Institute of Technology

DOI:

10.21203/rs.3.rs-19147/v1

\section{SUBJECT AREAS}

Materials Engineering

\section{KEYWORDS}

Functionally Graded Material, V-Reps, V-Models, Finite Cell Method, Direct

Simulation, Additive 24 Manufacturing, Homogenization 
Abstract

This paper proposes a computational methodology that allows a direct numerical simulation of heterogeneous/functionally graded materials based on V-reps/V-models and the Finite Cell Method (FCM). The FCM is an embedded domain approach that employs higher-order finite elements. The basic idea is to embed a complex geometric model into a fictitious domain that is trivial to mesh. The complexity of the geometry is then recaptured by an adapted precise numerical integration scheme for the elements cut by the boundary. For this, only a robust point inclusion test is required, which can be provided by various Computer-Aided Design (CAD) models. V-rep is a geometric modeling framework that represents the entire volume based on tri-variate B-Splines. Consequently, not only a point inclusion test is provided - but also the possibility to represent and model the interior domain. This allows to apply functionally graded material based on the tri-variate basis functions. These material parameters can then be regained during the simulation with an adapted point inclusion test.

The potential of the proposed method especially in the context of additive manufacturing is demonstrated by several numerical examples.

\section{Full Text}

Due to technical limitations, full-text HTML conversion of this manuscript could not be completed. However, the manuscript can be downloaded and accessed as a PDF.

\section{Figures}
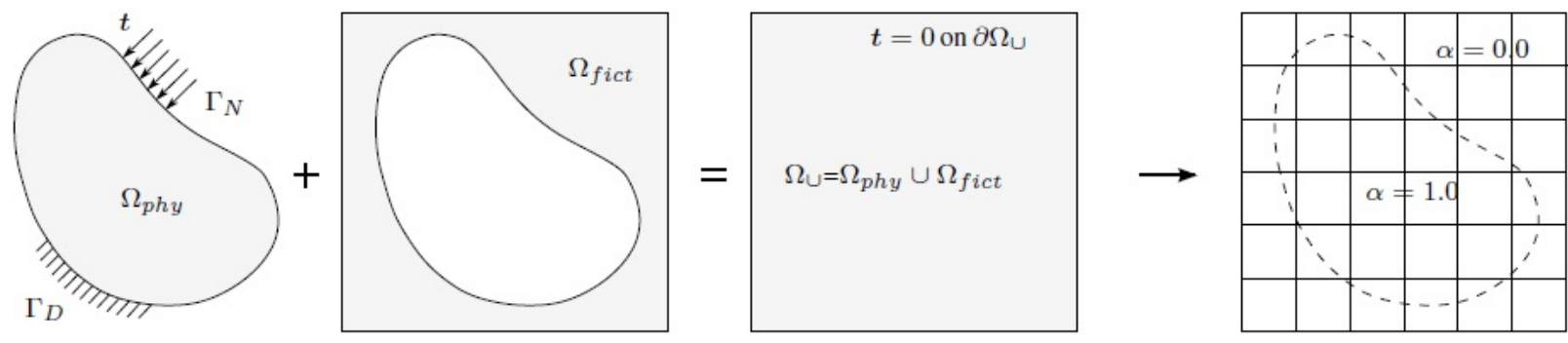

Figure 1

The concept of the finite cell method (taken from [35]). 


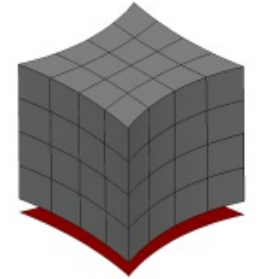

(a)

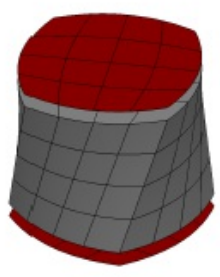

(b)

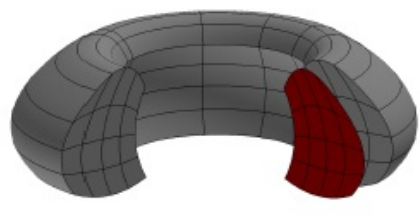

(c)

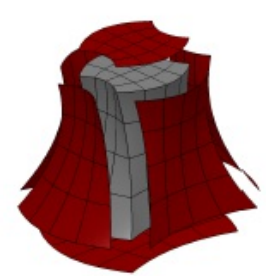

(d)

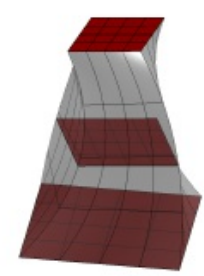

(e)

Figure 2

High-level primitives: (a) extrusion, (b) ruled solid, (c) volume of revolution, (d) boolean sum, and (e) sweep/loft.

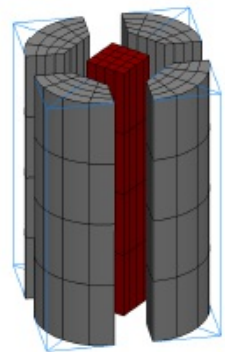

(a)

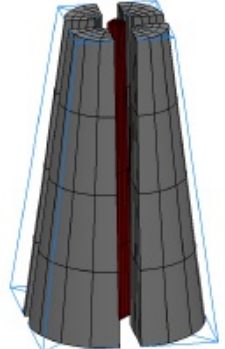

(b)

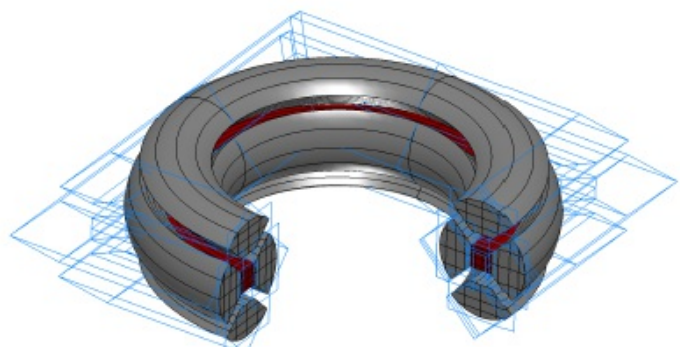

(c)

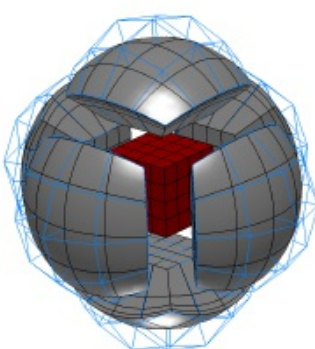

(d)

Figure 3

Non-singular primitives composed of trivariate B-splines: (a) A cylinder is composed by five extruded solids, whereas (b) a cone is composed of five ruled solids. (c) A torus is constructed using five solids of revolution, and (d) a sphere is composed of six ruled solids and one cuboid in its center. 

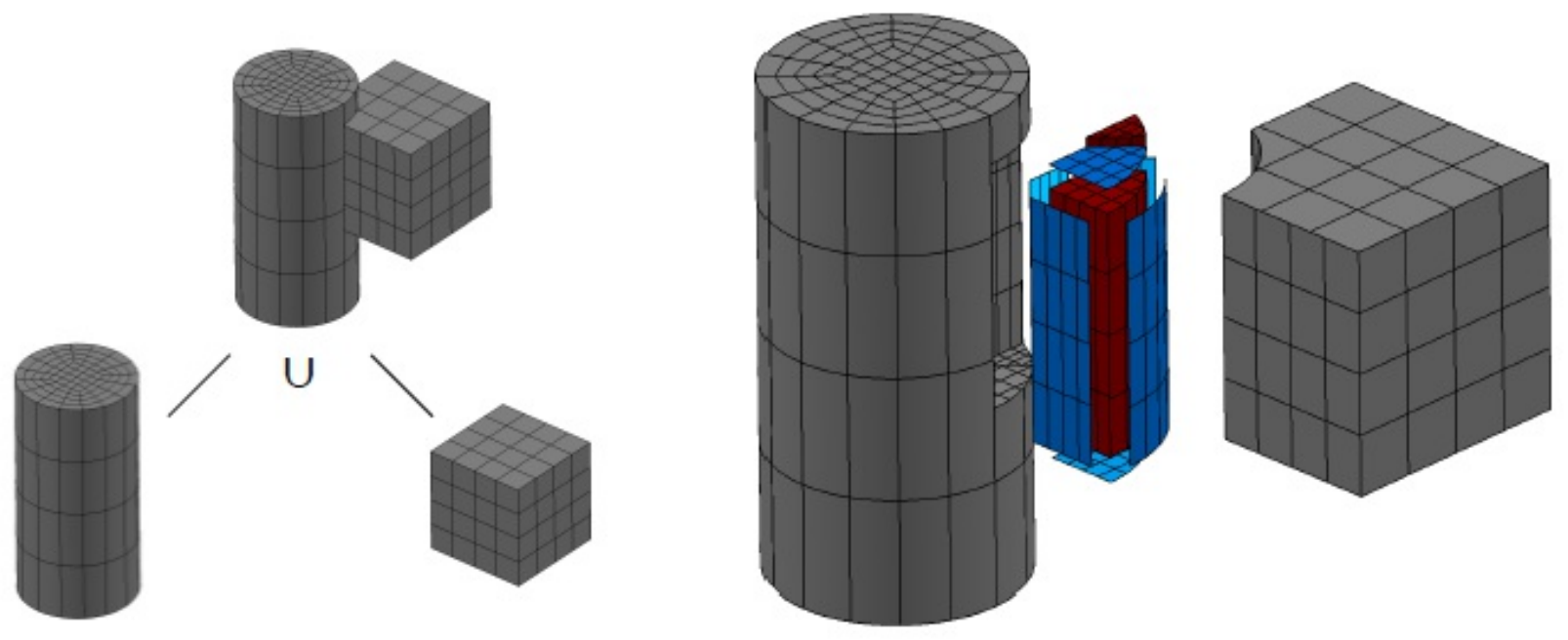

Figure 4

V-Model created as the union of a trivariate cuboid and a trivariate, non-singular cylinder. The inter-sected volume yields two V-cells (marked in red) which are constructed with the trimming surfaces (highlighted in blue). 


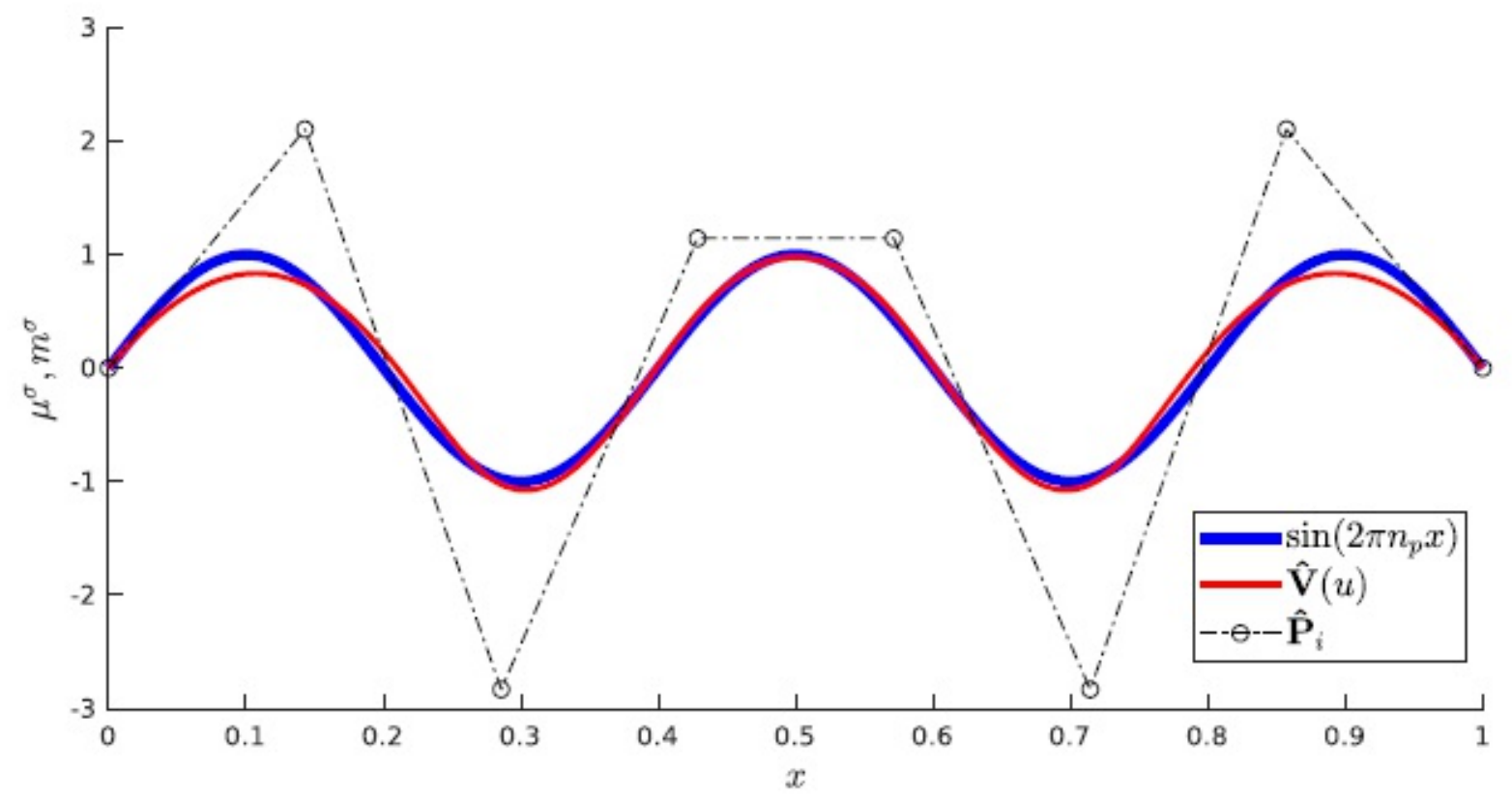

Figure 5

One-dimensional least squares approximation of a hypothetical sinusoidal material function $m \sigma(x)=\sin (2 \pi n p x)$, with $n p=2.5$ being the number of periods, yields the material 'coordinates' $\mu$ i $\sigma$. Note that the rather large deviation between the curves comes from the fact that the location (i.e. $x$-coordinate) of material control points is fixed. 


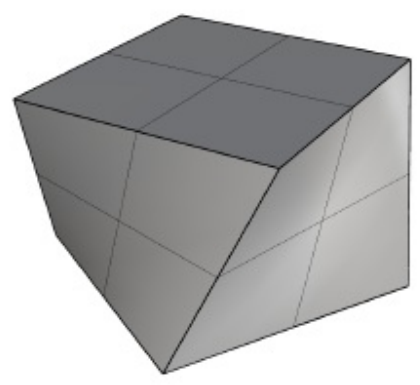

(a) Ruled base volume

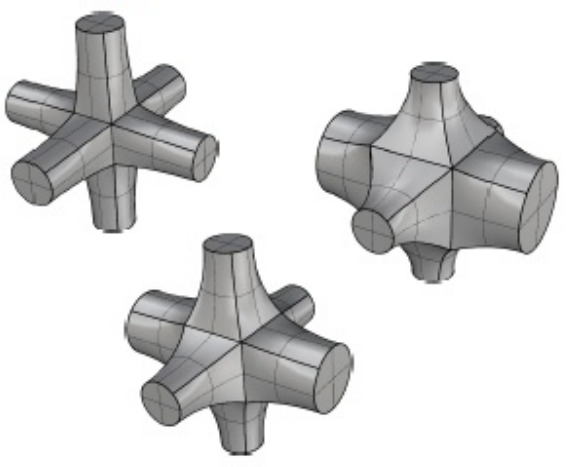

(b) Unit tiles

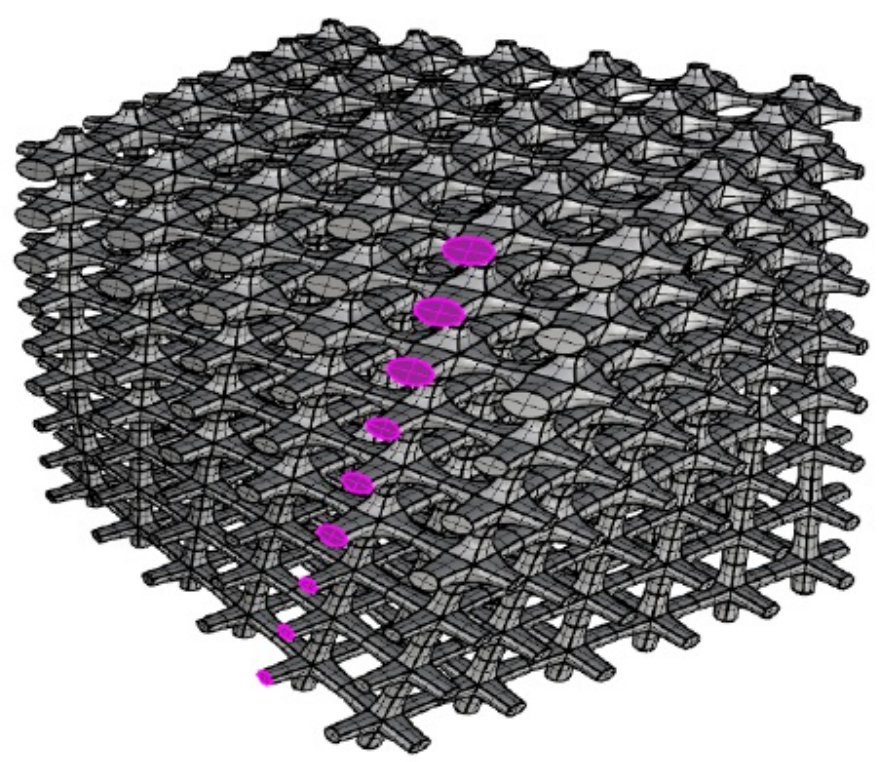

(c) microstructure

Figure 6

Functionally graded microstructure: (b) Three different anisotropic tiles, with a changing

stiffer direction, are used to tile (a) a rotating ruled volume. (c) The entire resulting microstructure exhibits a continuously changing anisotropic stiffness. 


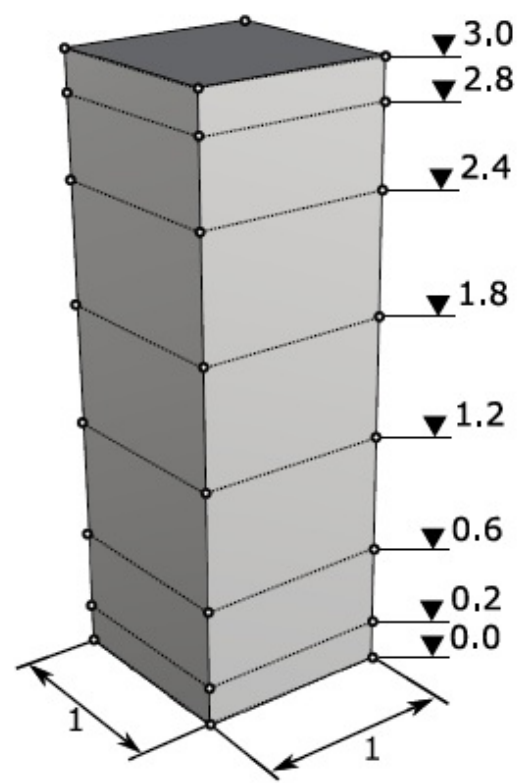

(a)

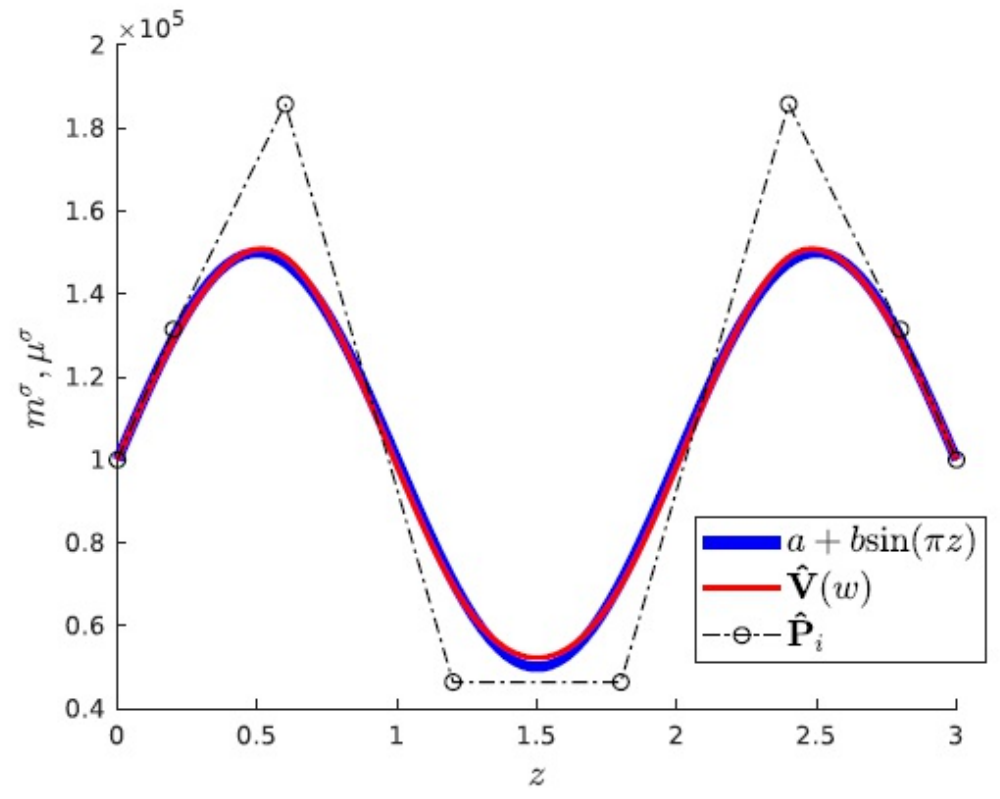

(b)

Figure 7

(a) Dimensions of the cuboid. (b) Least squares ï ttting of $E(z)$ yielding the material coordinates ÂuiE . 


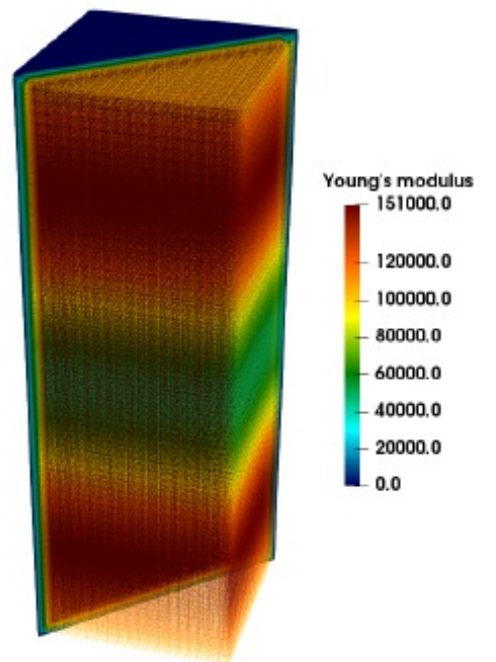

(a)

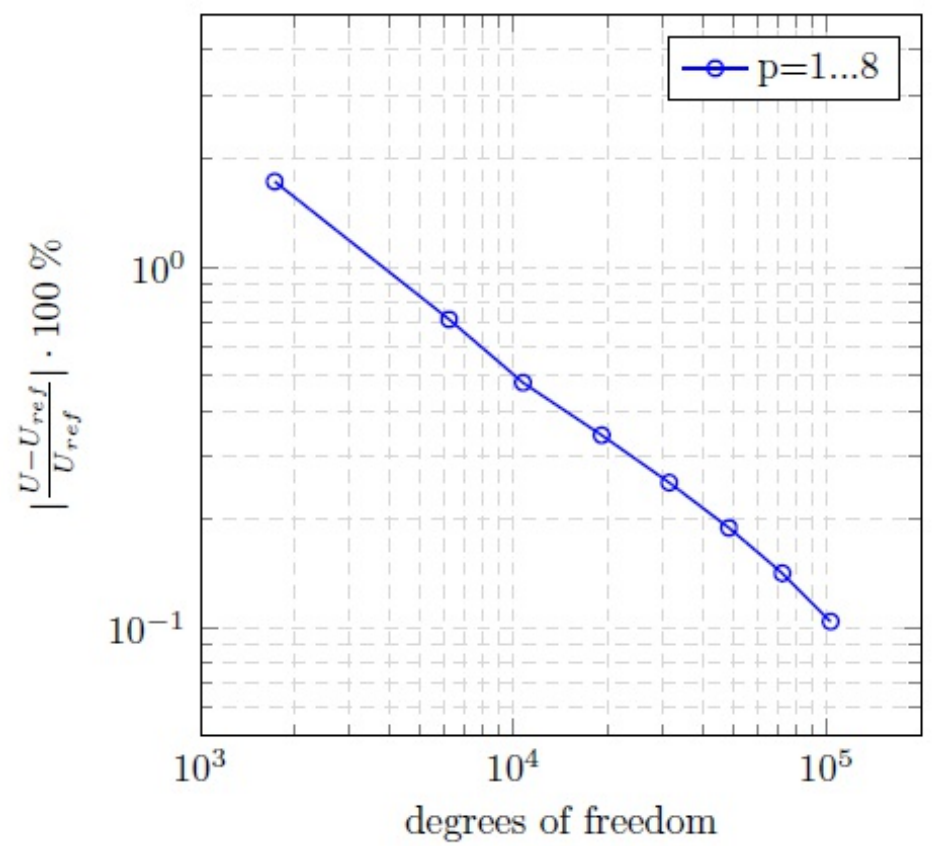

(b)

Figure 8

(a) Youngâ $€^{\mathrm{TM}} \mathrm{S}$ modulus evaluated on integration points inside the cuboid and in the ï ctitious domain.(b) Relative error in the strain energy for polynomial degrees $p=1 \ldots 8$. 


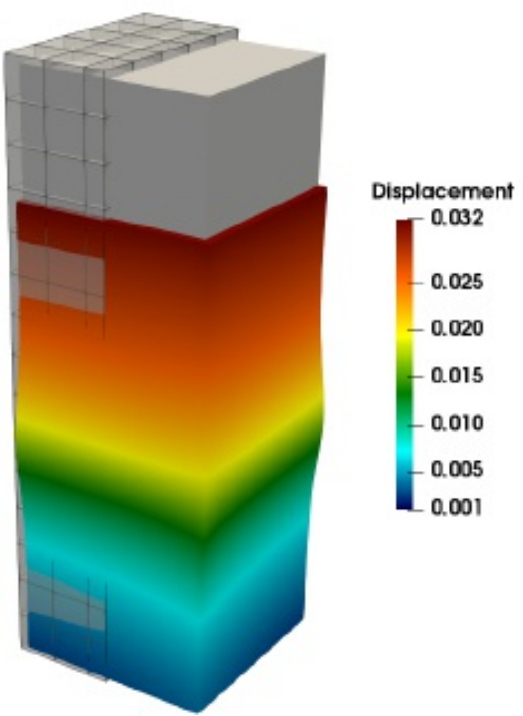

(a)

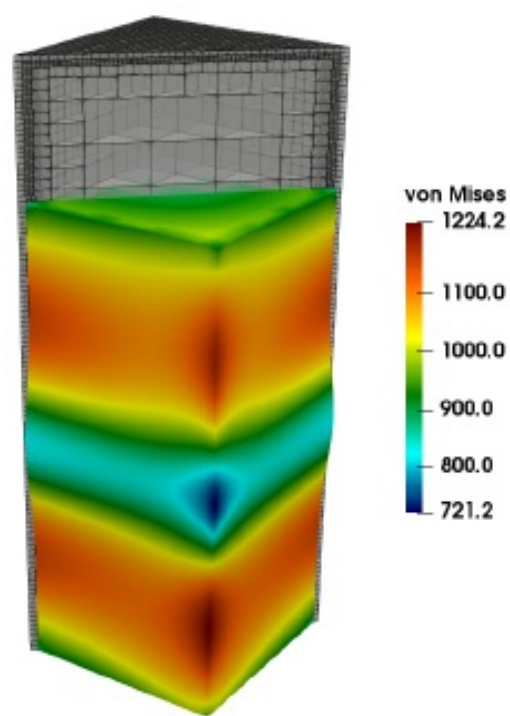

(b)

Figure 9

(a) Displacements warped around the undeformed cuboid (grey block) embedded into the finite cell mesh. (b) Von Mises stresses and integration mesh. The deformation is scaled by a factor of 20.

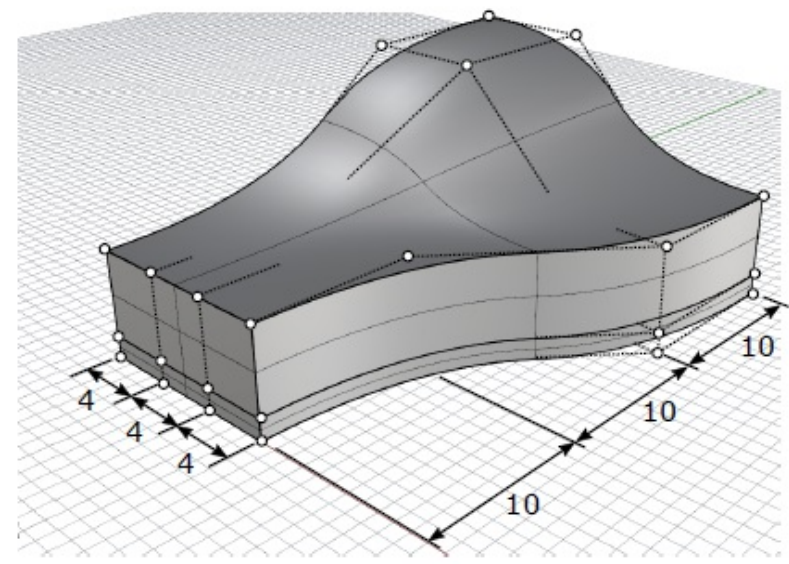

(a)

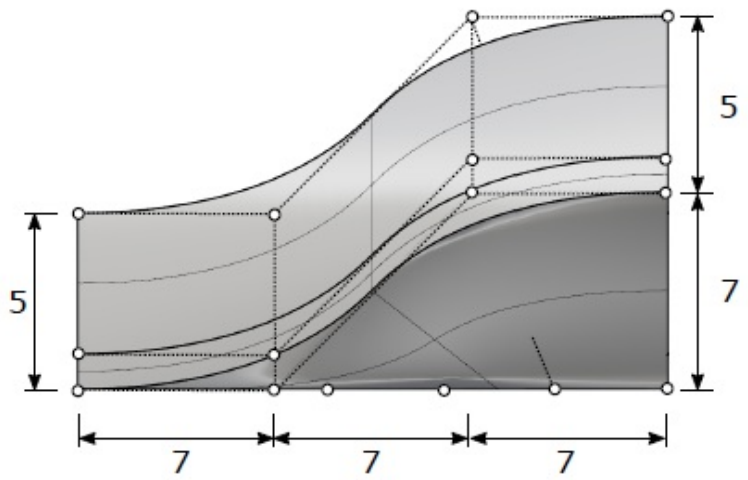

(b)

Figure 10

Model dimensions (in $\mathrm{cm}$ ) and control point mesh of the discontinuous tile in (a) isometric view, and (b) from the back side. 


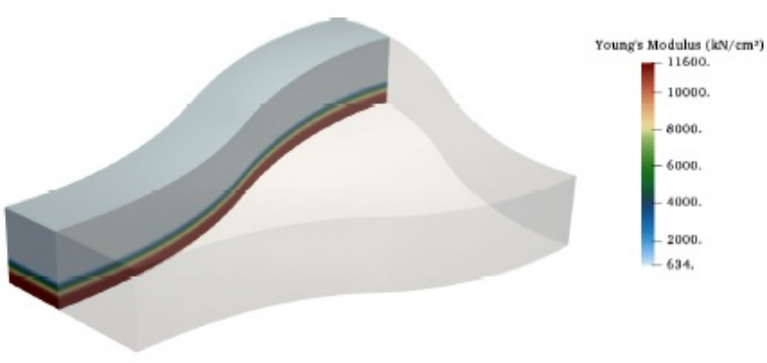

(a)

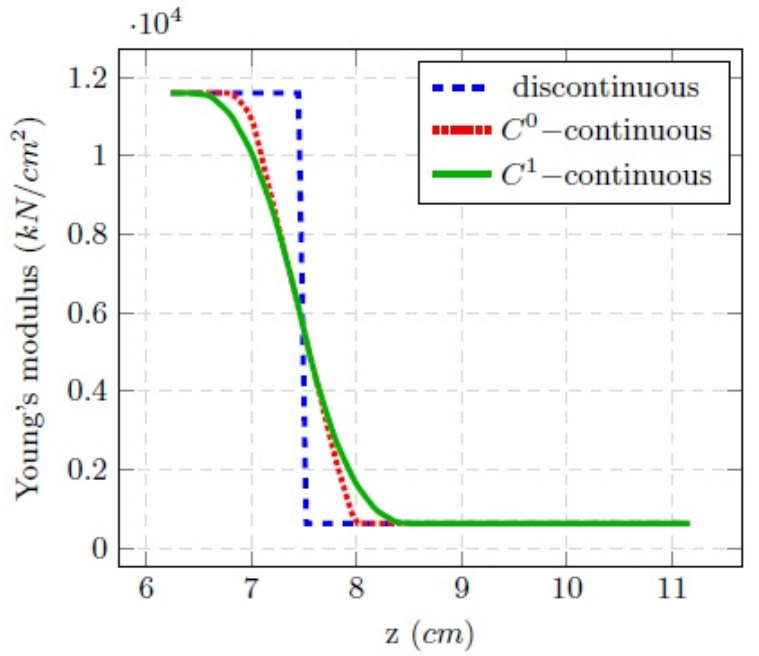

(b)

Figure 11

Material distribution of the Young's modulus (a) inside the C0-continuous tile and (b) plotted at $x=5 \mathrm{~cm}, y=25 \mathrm{~cm}$ over the thickness.

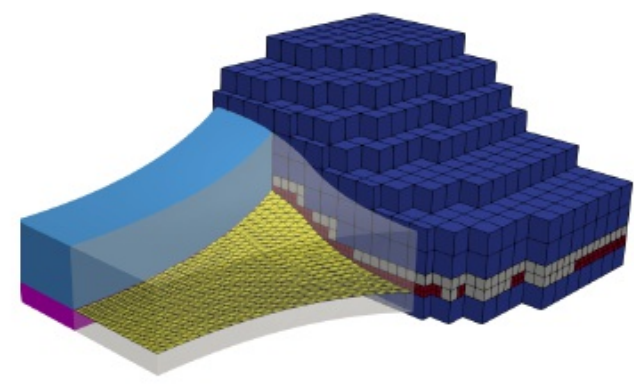

(a)

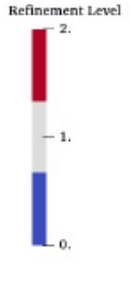

Figure 12

Discretizations of $(a)$ the discontinuous tile: The mesh is refined twice around the coupling surface (yellow), which divides the upper (light blue) and lower domain (purple). (b) The C0-continuous tile: The FCM mesh is refined once in the transition zone (cells in blue are unrefined, and cells in red are refined once). The grey mesh in the background corresponds to the octree for the integration. The $\mathrm{C} 1$-continuous tile is meshed and refined analogously. 


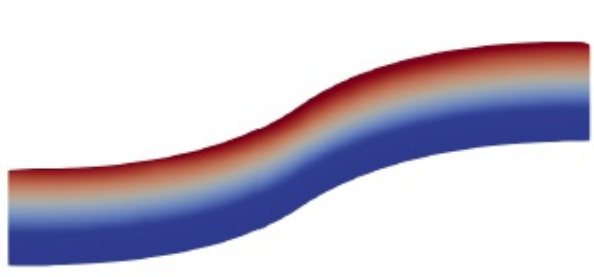

(a)
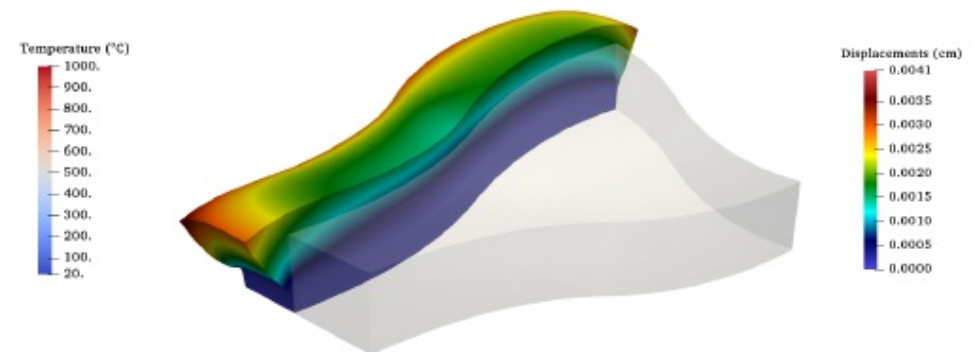

(b)

Figure 13

C0-continuous tile: (a) Temperature distribution and (b) displacements warped by a scaling factor of 1000 .

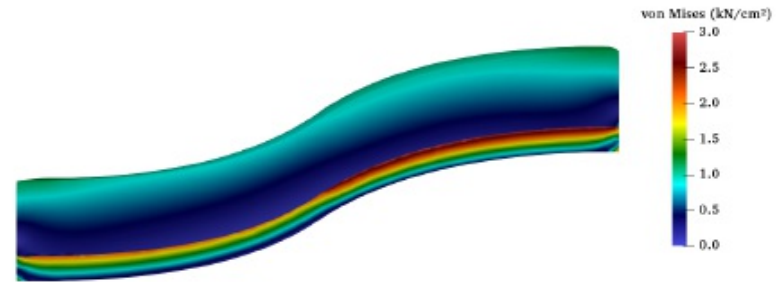

(a)

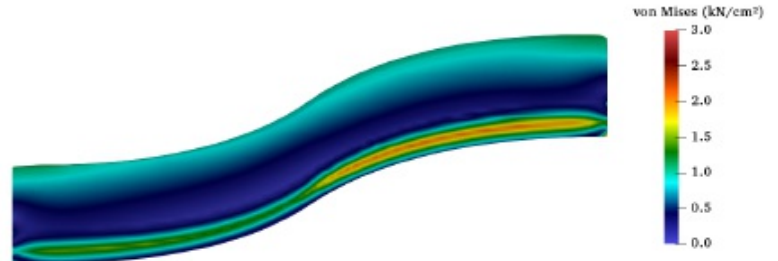

(b)

Figure 14

Von Mises stresses of the (a) discontinuous and (b) COâ^'continuous thermal shielding tile. The stress distribution of the Clân'continuous tile looks very similar to the C0ân'continuous tile. 


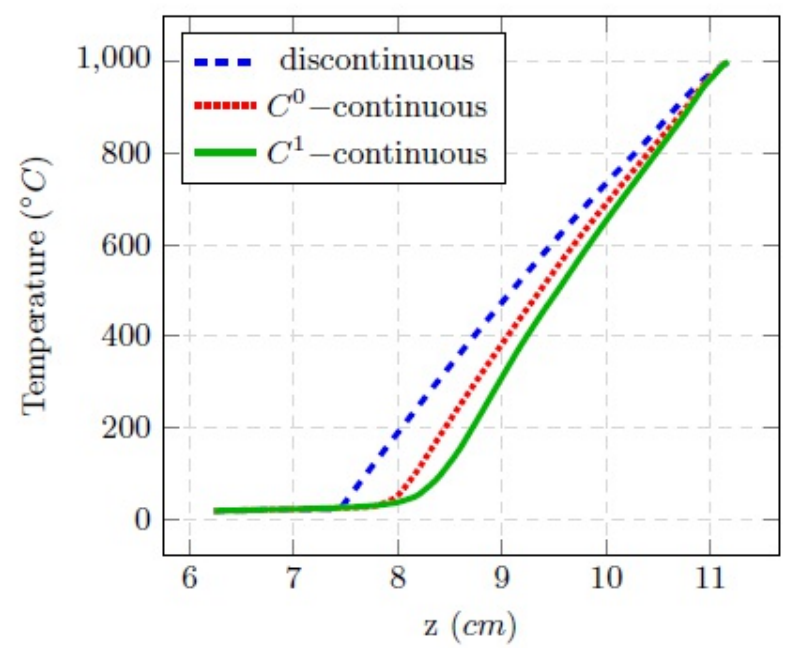

(a)

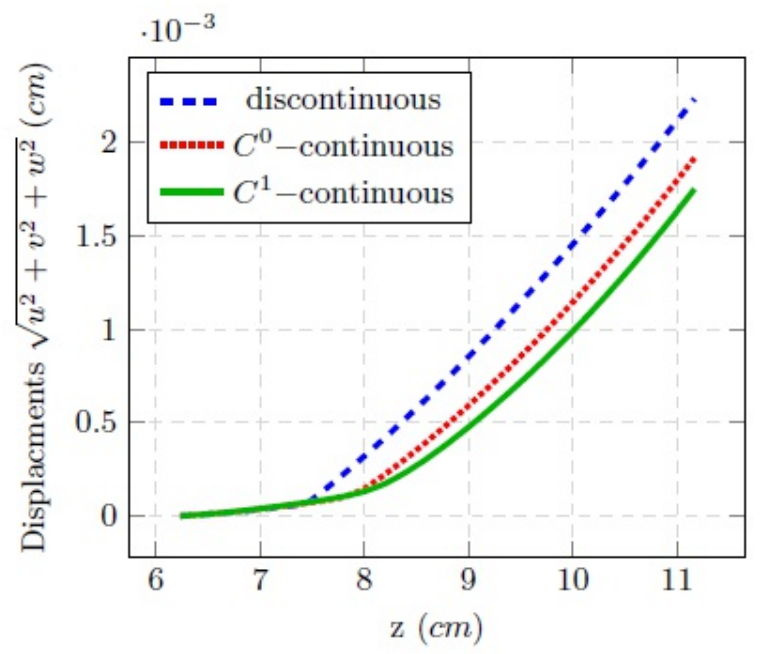

(b)

Figure 15

Comparison of (a) the temperature and (b) the displacements of the discontinuous and continuous tiles at $x=5 \mathrm{~cm}, y=25 \mathrm{~cm}$ over the thickness.

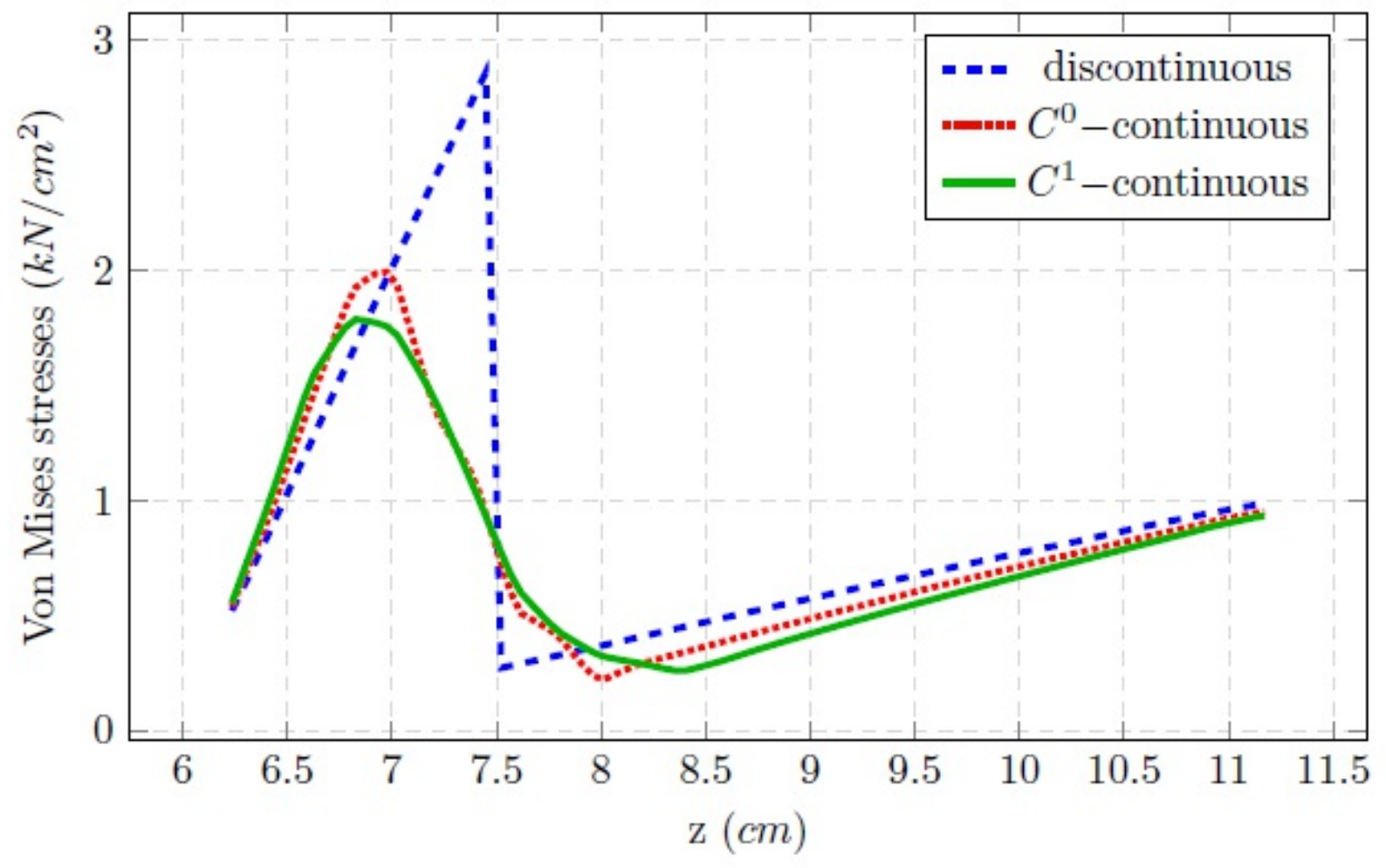

Figure 16

Comparison of the von Mises stresses of the discontinuous and continuous tiles at $x=5 \mathrm{~cm}$, $y=25 \mathrm{~cm}$ over the thickness. 


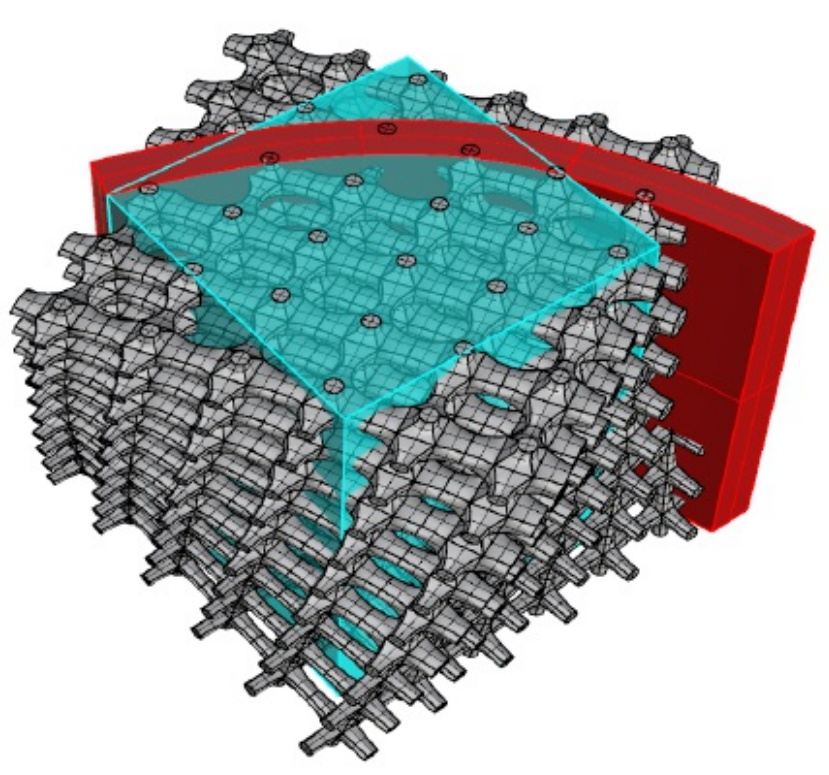

(a)

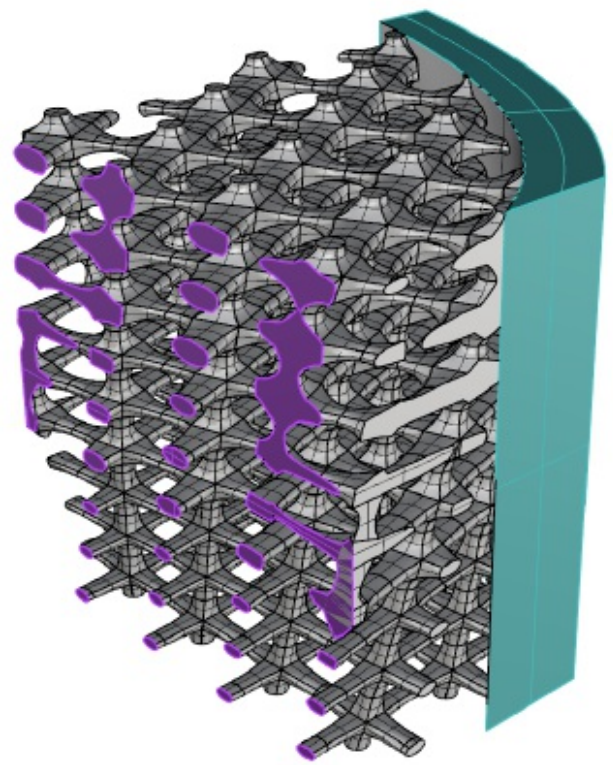

(b)

Figure 17

(a) Selection of the computational domain (turquoise). An outer shell (red) is embedded into

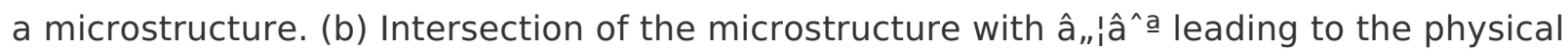
domain â,,'phy. Boundary conditions are applied on the highlighted intersection surfaces.

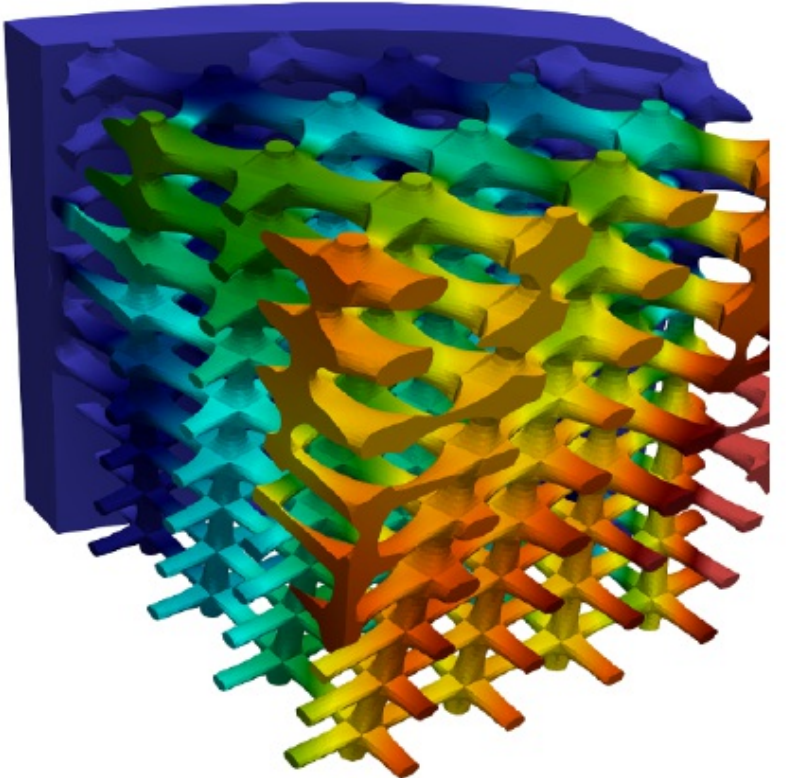

(a)

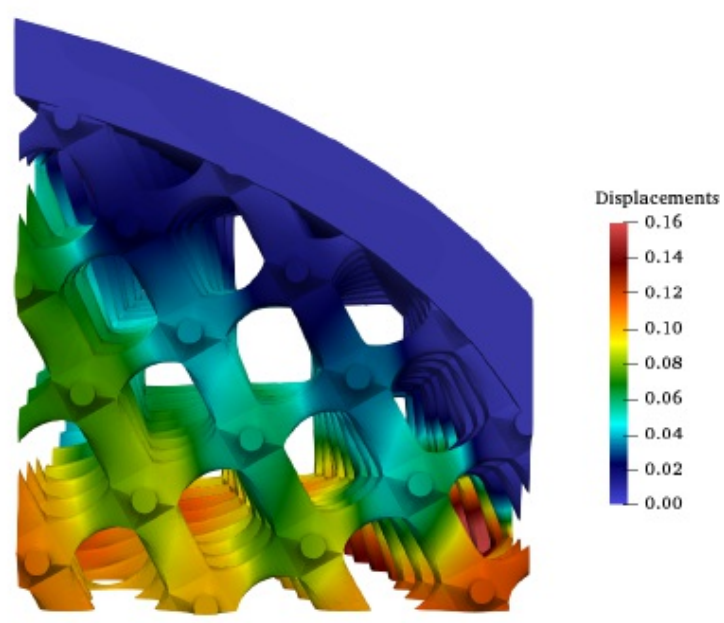

(b)

Figure 18

Displacements in (a) isometric view and (b) from the top. 


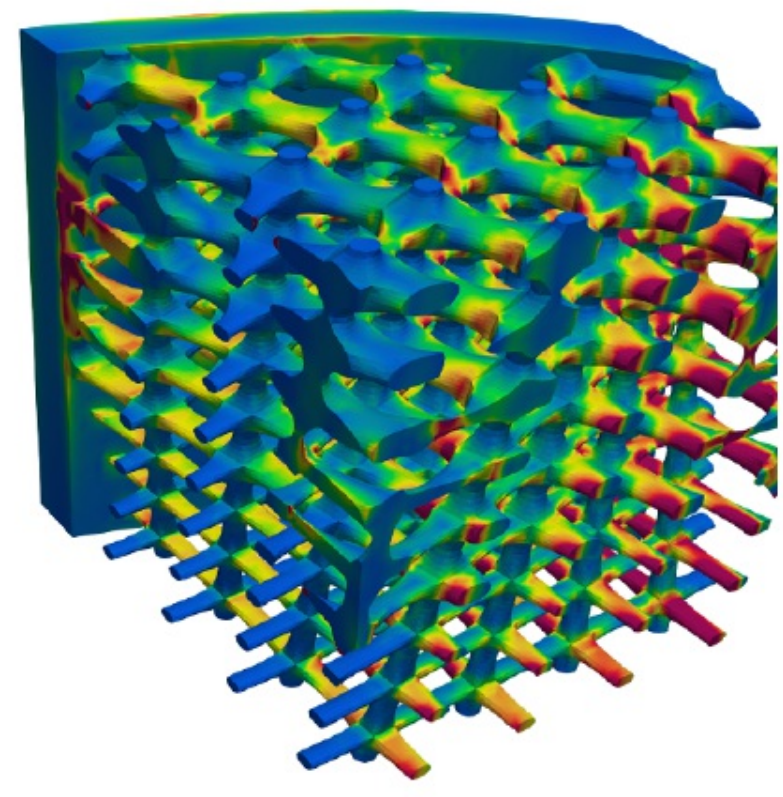

(a)

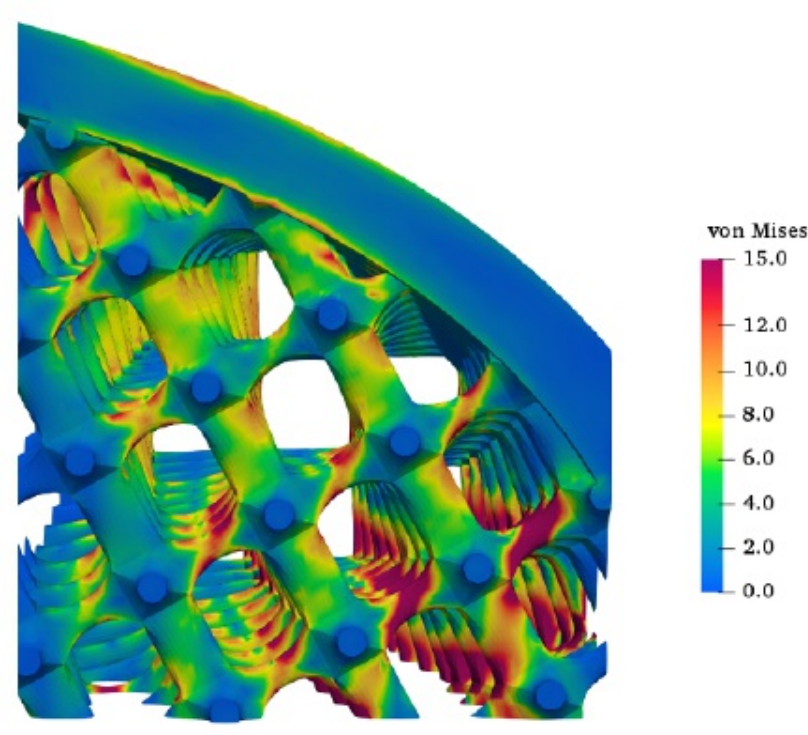

(b)

Figure 19

Von Mises stresses in (a) isometric view and (b) from the top.

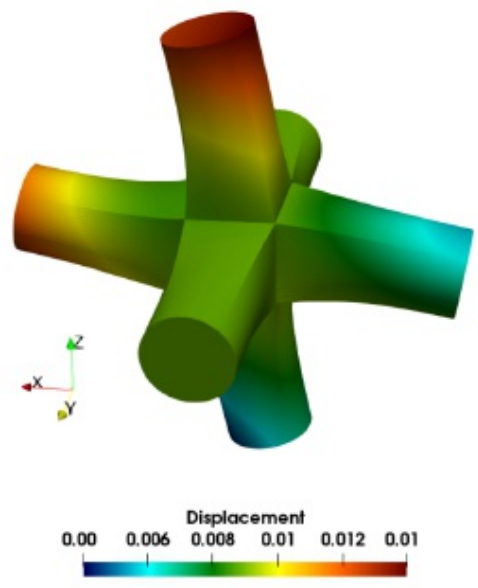

(a) Tile 1

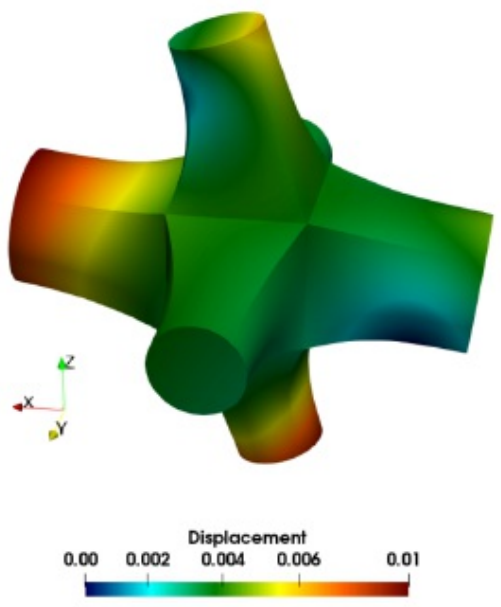

(b) Tile 2

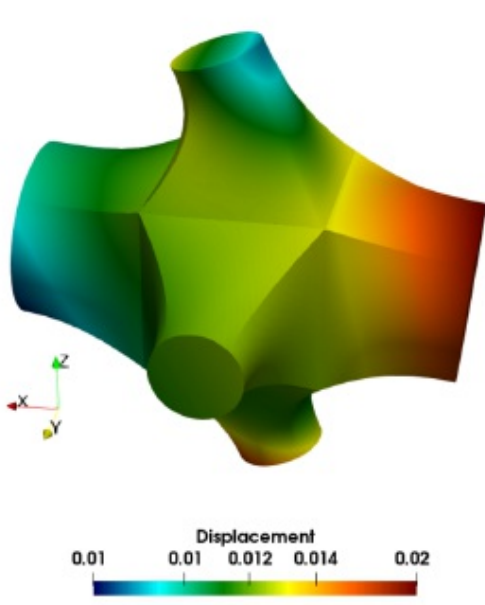

(c) Tile 3

Figure 20

Displacement field of the warped tiles with a scale factor $s=10$. 


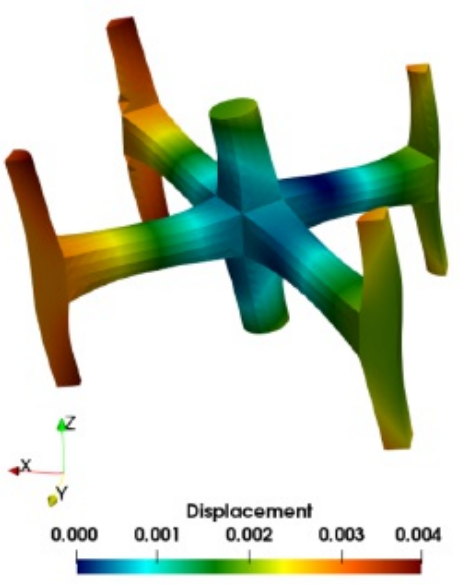

(a) Tile 1

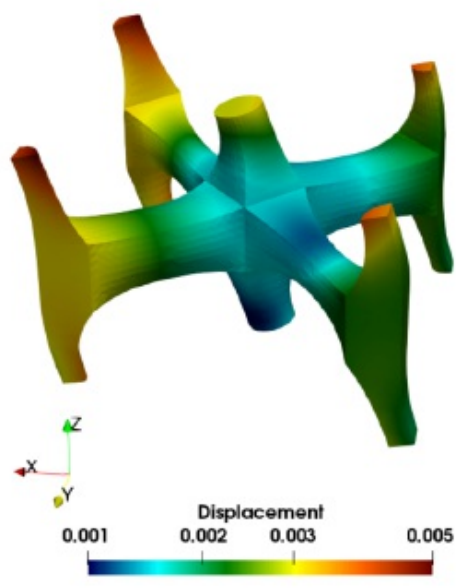

(b) Tile 2

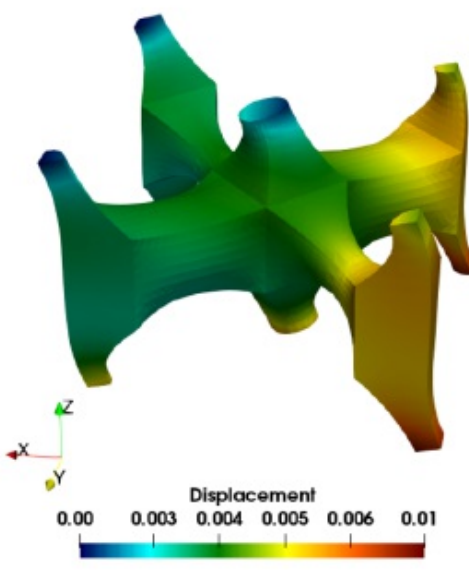

(c) Tile 3

Figure 21

Displacement field of the warped rotated tiles with a scale factor $s=10$.

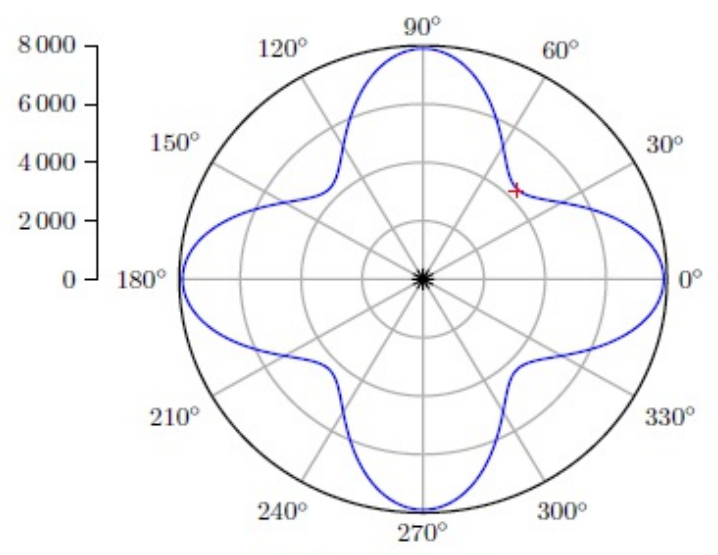

(a) $C_{11},(M P a)$

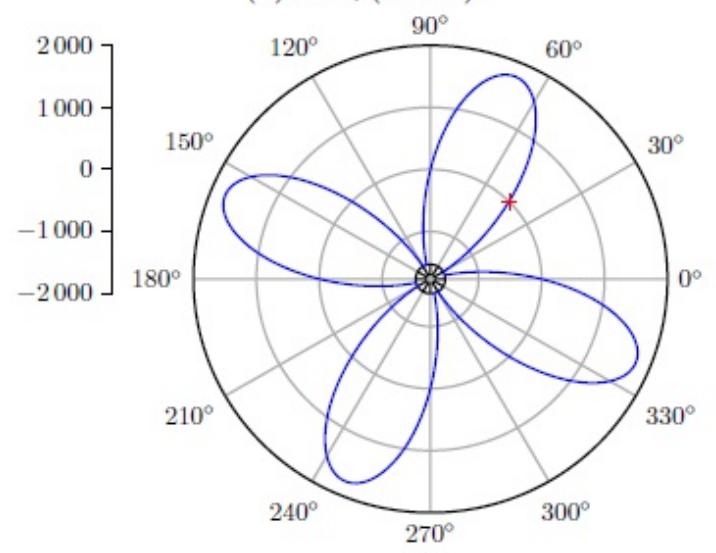

(c) $C_{14},(M P a)$

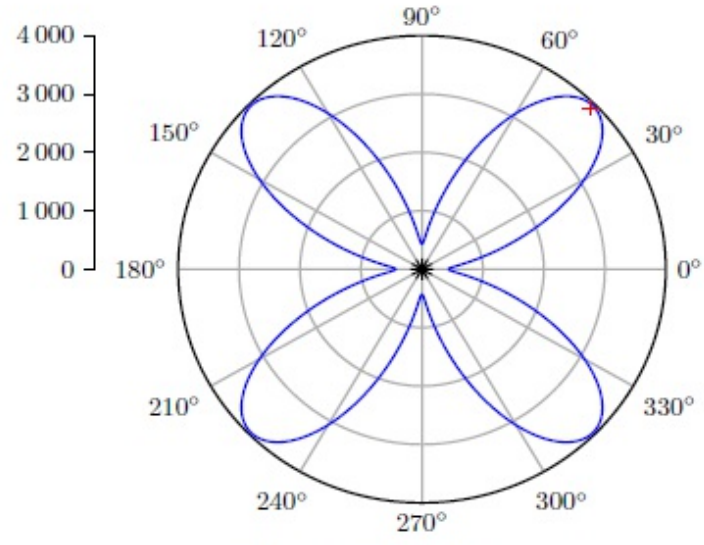

(b) $C_{12},(\mathrm{MPa})$

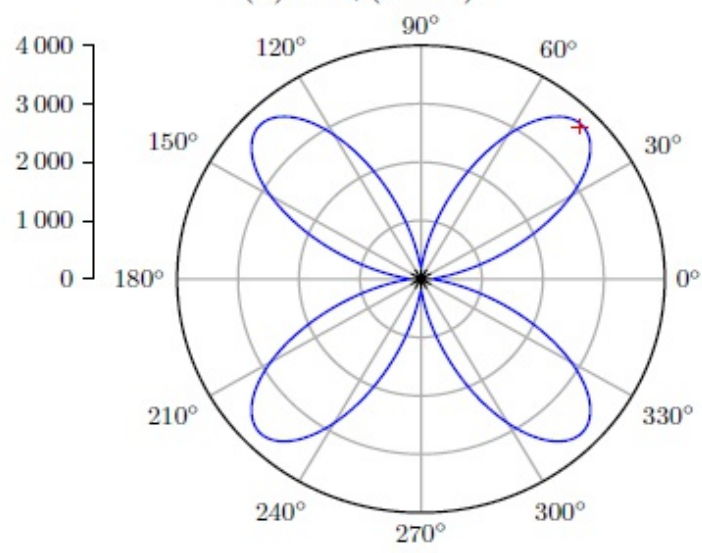

(d) $C_{44},(\mathrm{MPa})$

Figure 22

Independent elastic constants for tile 1 under rotation around the $z$-axis. 


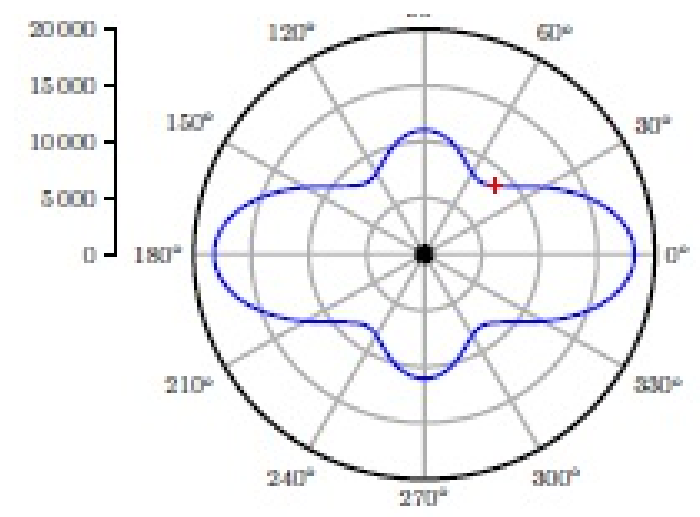

(a) $C_{11},(M P a)$

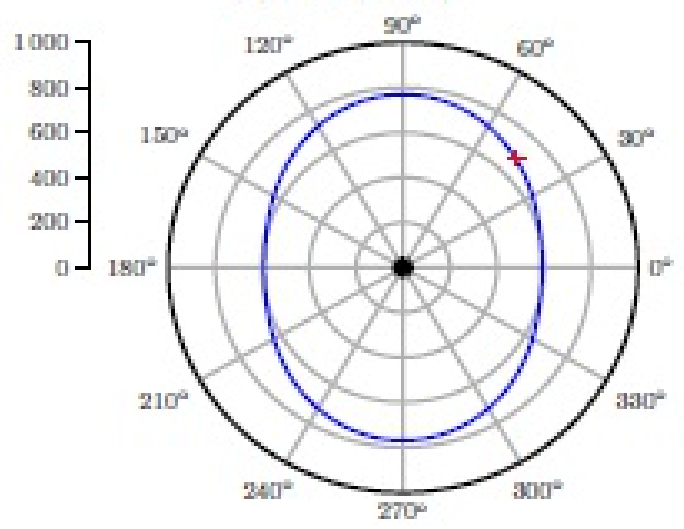

(c) $\mathrm{C}_{\mathrm{6̆}},(\mathrm{MPa})$

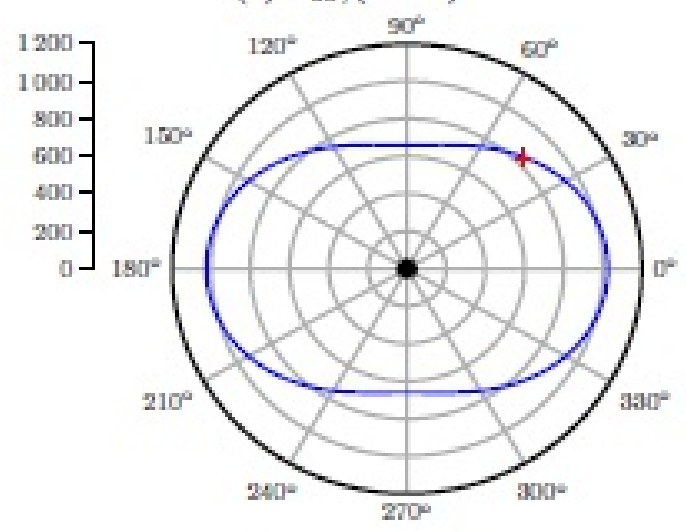

(e) $C_{13},(M P a)$

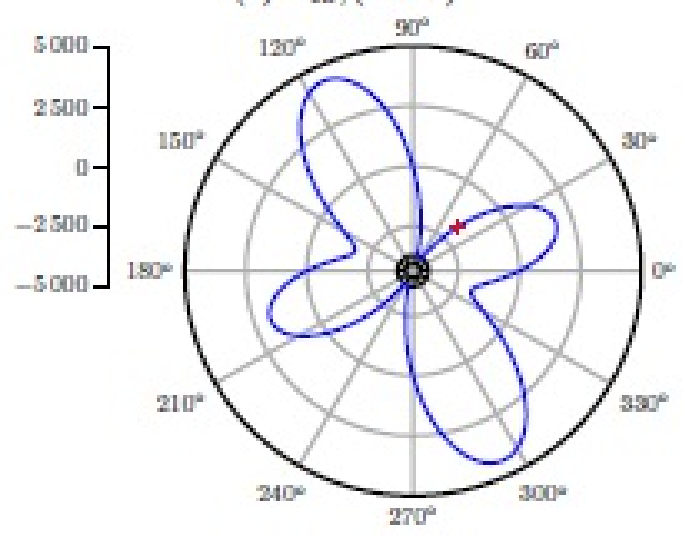

(g) $\mathrm{C}_{24},(\mathrm{MPa})$

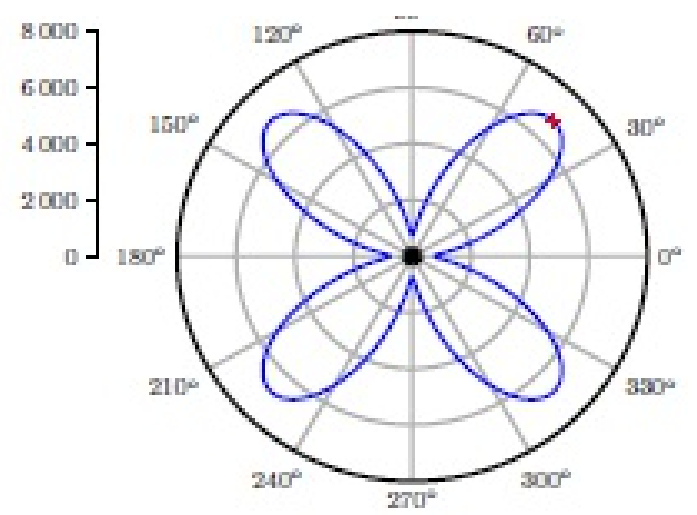

(b) $C_{44},(M P a)$

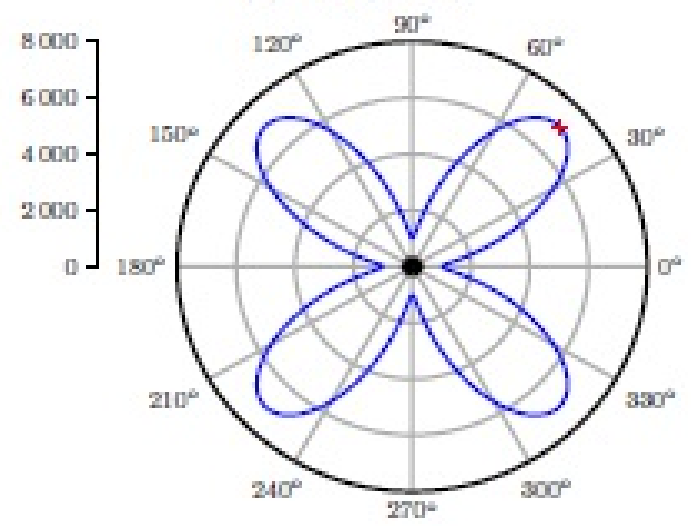

(d) $C_{12},(M P a)$

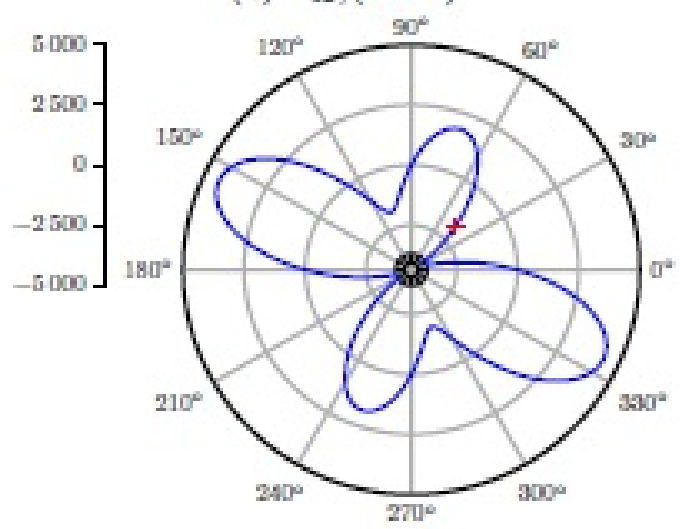

(f) $C_{14},(\mathrm{MPa})$

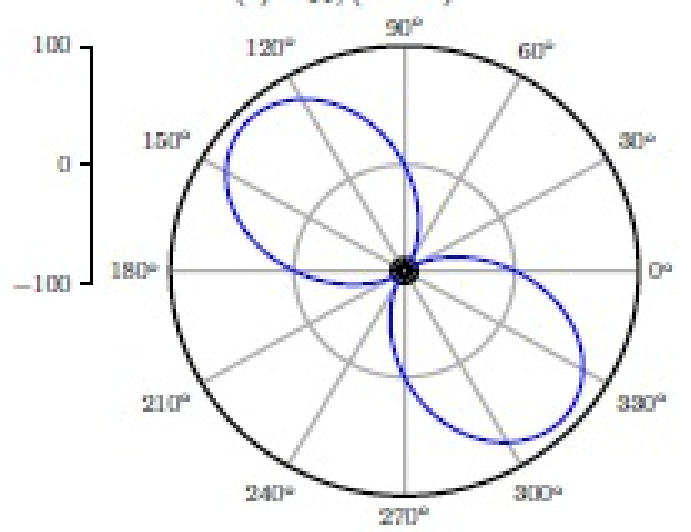

(h) $C_{\mathrm{sE}},(\mathrm{MPa})$

Figure 23 
Independent elastic constants for tile 2 under rotation around the zâ^’axis.

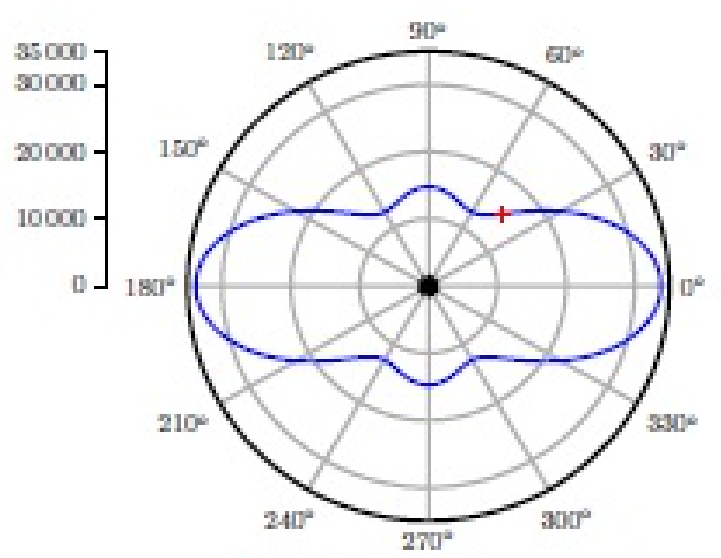

(a) $C_{11},(M P a)$

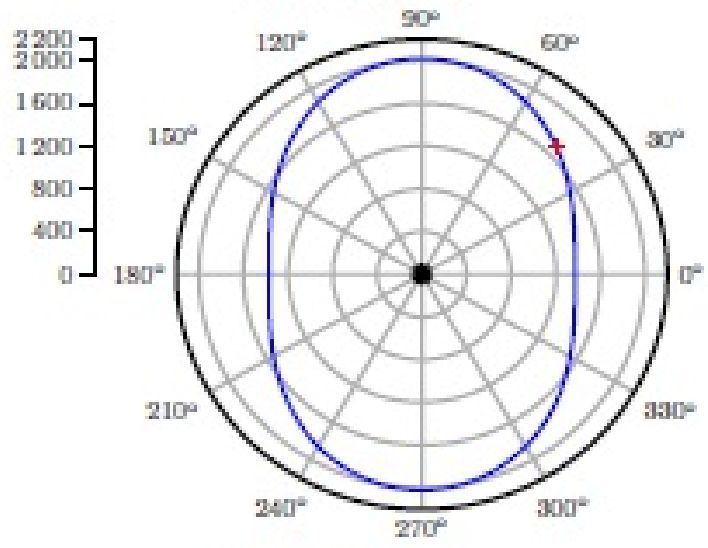

(c) $C_{\mathrm{s}},(\mathrm{MPa})$

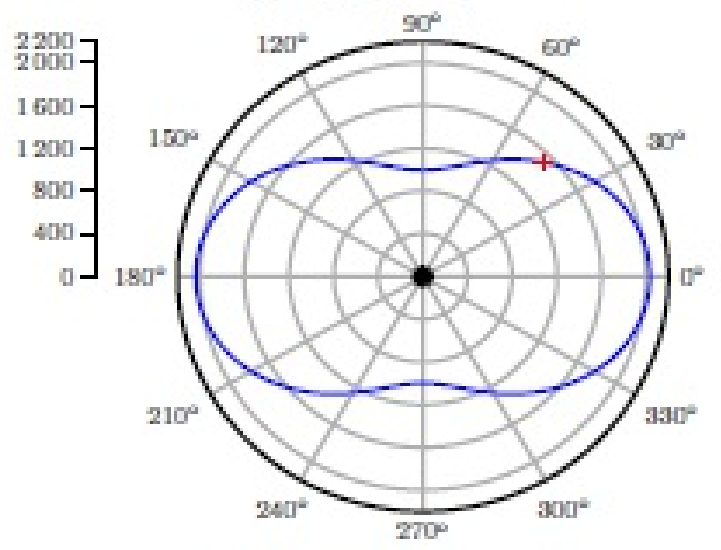

(e) $C_{13},(M P a)$

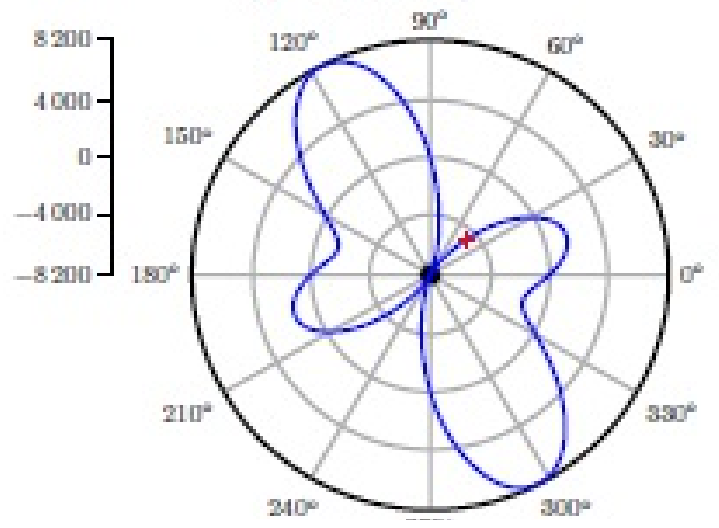

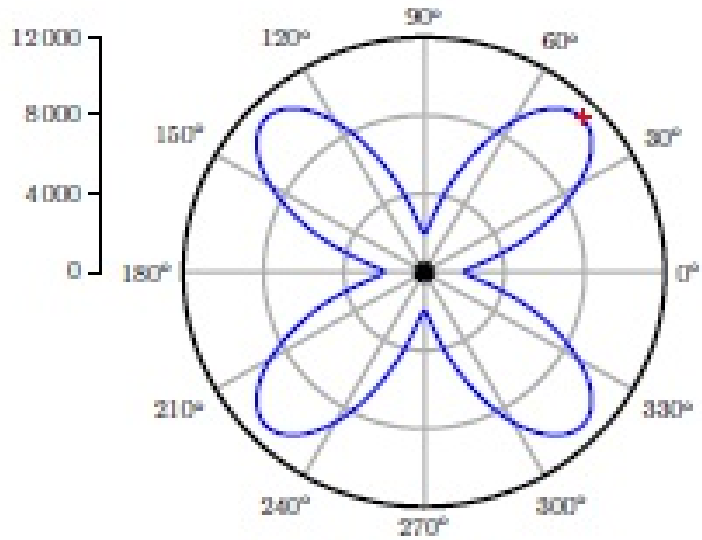

(b) $\mathrm{C}_{44},(\mathrm{MPa})$

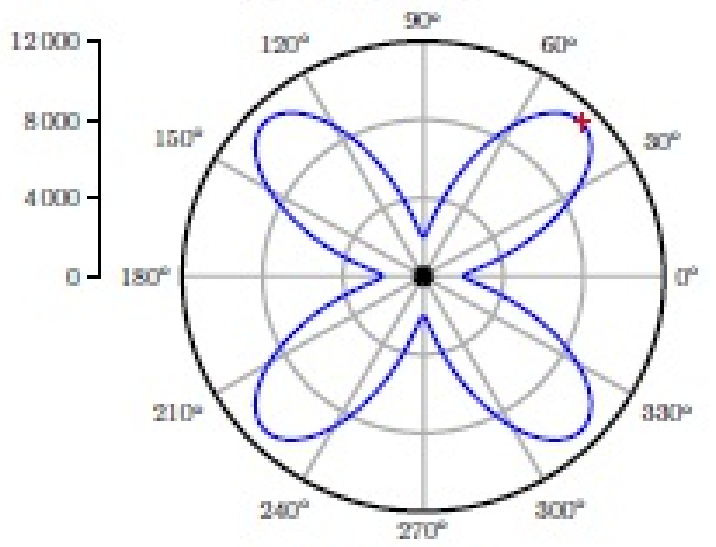

(d) $C_{12},(M P a)$

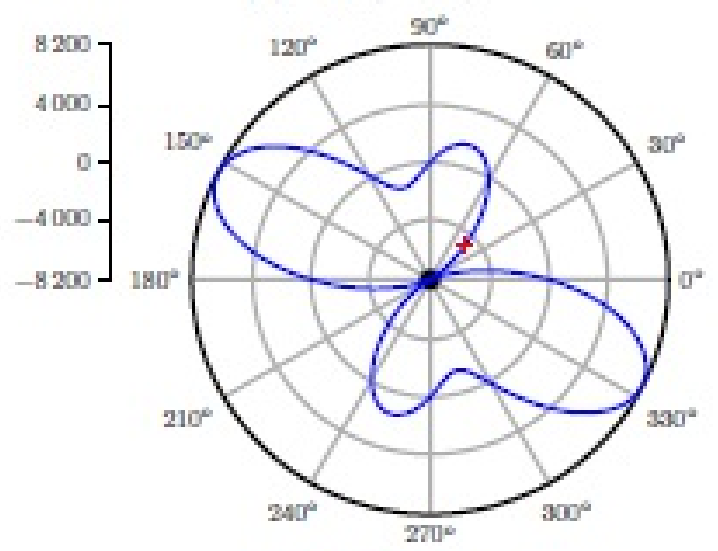

(f) $\mathrm{C}_{14},(\mathrm{MPa})$

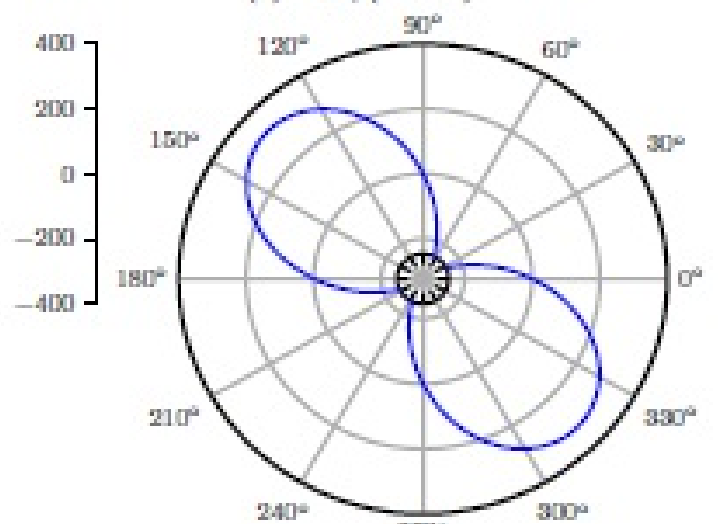


Figure 24

Independent elastic constants for tile 3 under rotation around the $z$-axis.

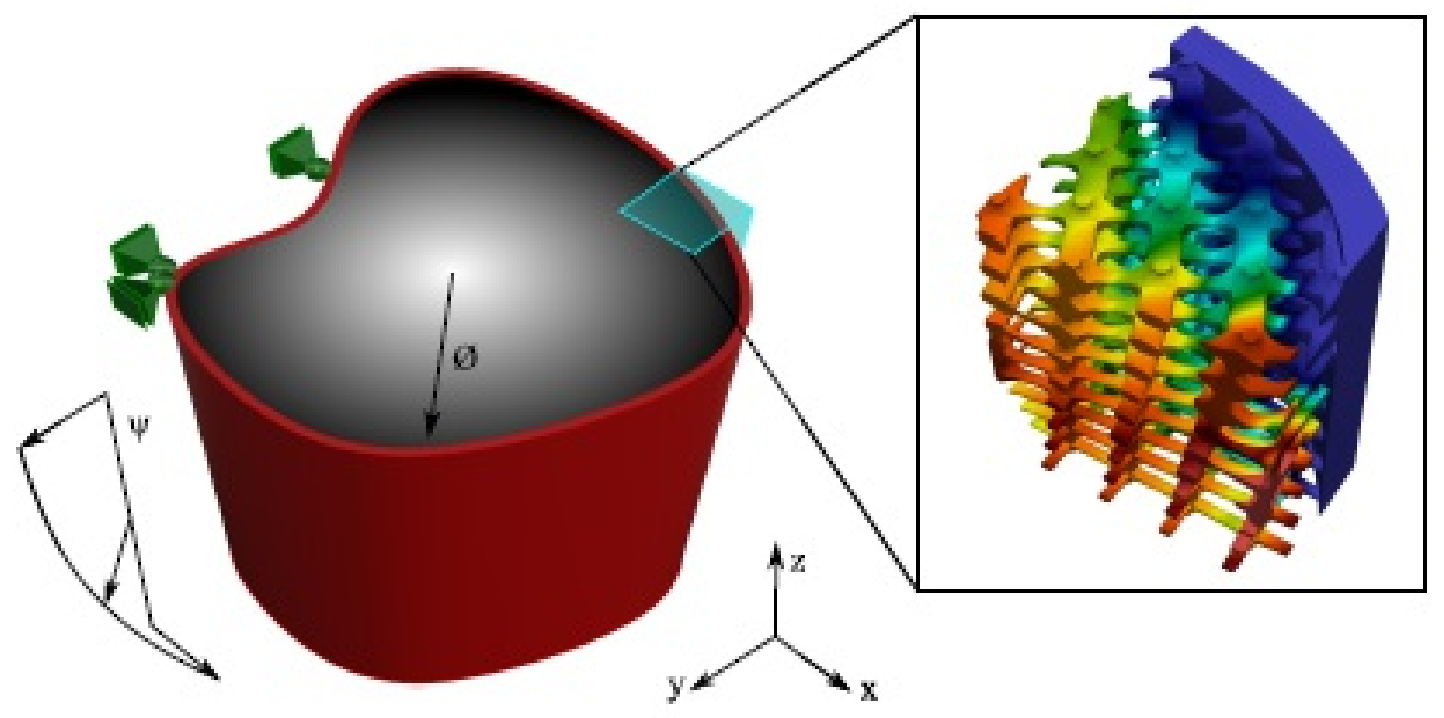

Figure 25

Structure consisting of a solid shell (red) and a homogenized microstructure (gray scale).

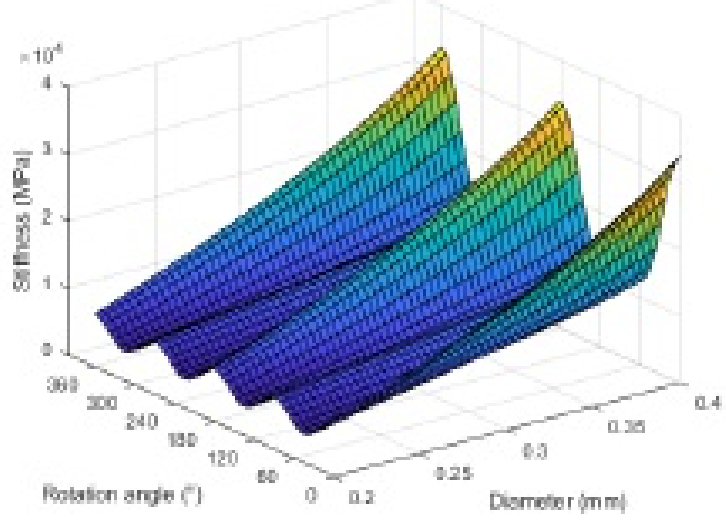

(a) $C_{11}$

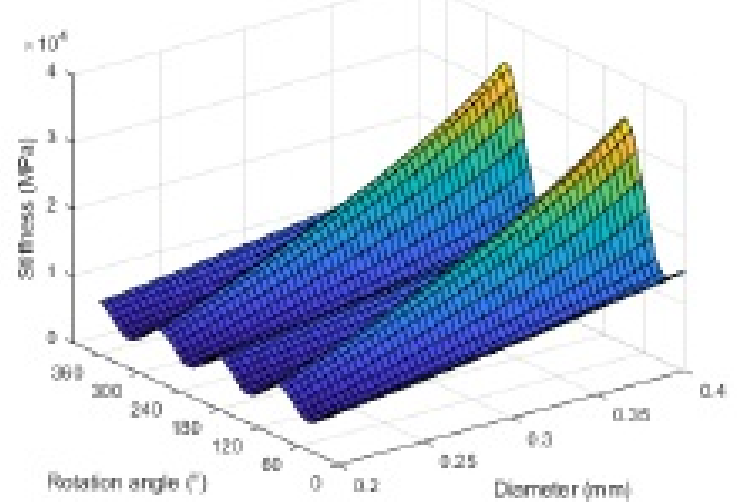

(b) $\mathrm{C}_{22}$

Figure 26

Spline based interpolation of the material coefficients C11 and C22. 


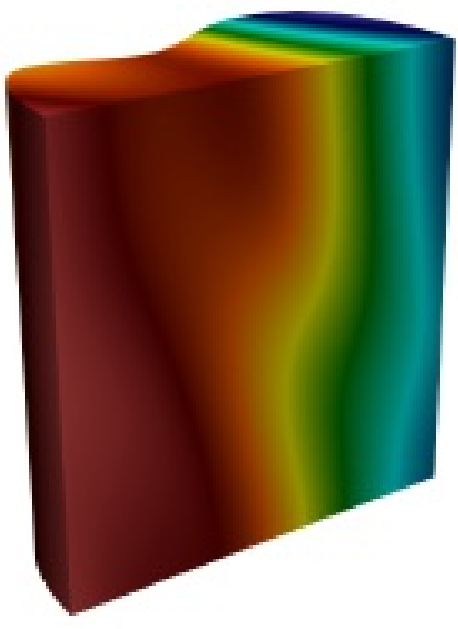

(a)

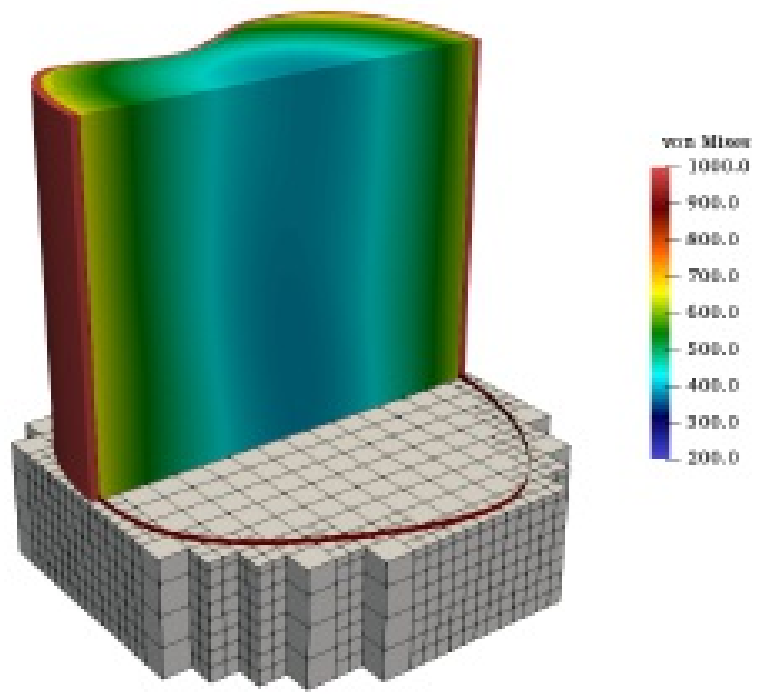

(b)

Figure 27

(a) Displacements in $\mathrm{x}$-direction and (b) von Mises stresses with the finite cell mesh.

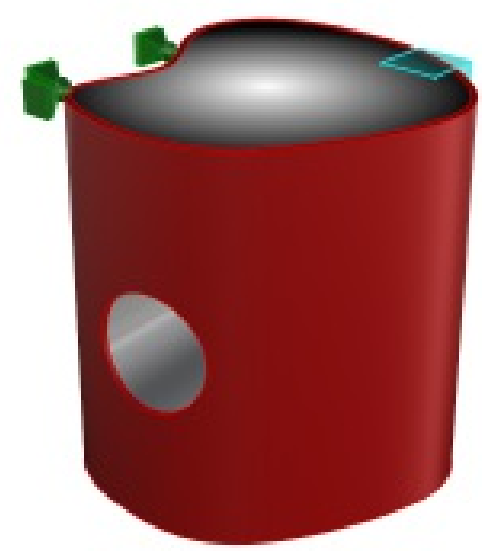

(a)

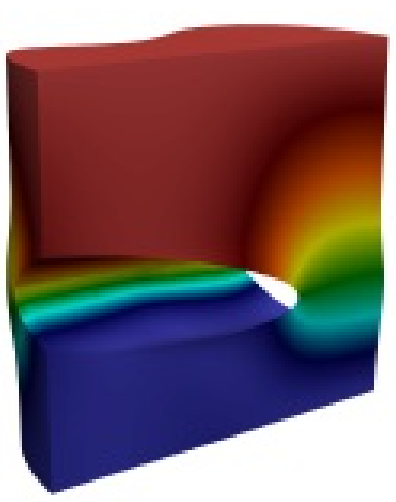

(b)

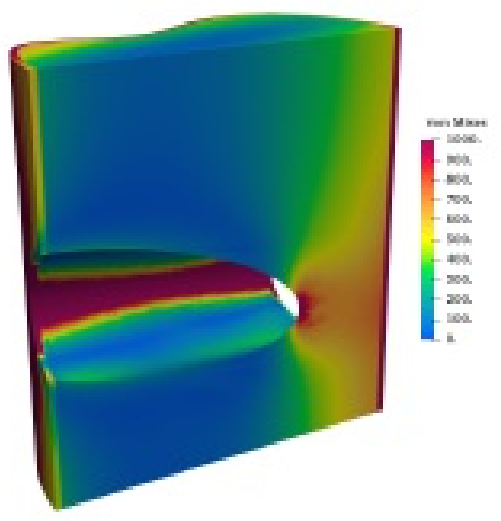

(c)

Figure 28

Structure with hole: (a) model, (b) displacements in $z$-direction (warped by a factor of $s=$ 2), and (c) von Mises stresses. 